\title{
Cysteine String Protein Is Required for Calcium Secretion Coupling of Evoked Neurotransmission in Drosophila But Not for Vesicle Recycling
}

\author{
Ravi Ranjan, Peter Bronk, and Konrad E. Zinsmaier \\ Department of Neuroscience, University of Pennsylvania School of Medicine, Philadelphia, Pennsylvania 19104-6074
}

The entire deletion of the cysteine string protein (CSP) gene causes a temperature-sensitive (ts) block of evoked neurotransmission in Drosophila. CSP has been found to interact in vitro with the clathrin-uncoating ATPase HSC70, suggesting a potential role of CSP in vesicle recycling. Using FM1-43 imaging, we analyzed whether the ts block of neurotransmission in csp mutants is caused by a defect in vesicle exocytosis or vesicle recycling. We determined that FM1-43-labeled synaptic boutons of $\operatorname{csp}$ mutant neuromuscular junctions fail to destain at $32^{\circ} \mathrm{C}$ after $\mathrm{K}^{+}$depolarization, and that $\mathrm{FM} 1-43$ dye uptake cannot be evoked by $\mathrm{K}^{+}$stimulation at $32^{\circ} \mathrm{C}$. However, when we stimulated dye uptake independent of depolarization by using black widow spider venom (BWSV), we observed endocytotic uptake of FM1-43. This suggests that endocytosis exhibits no primary ts defect. In addition, we found no ts defect of vesicle recycling at $32^{\circ} \mathrm{C}$ that would correlate with the ts block of neurotransmission. We also discovered that BWSV and the calcium ionophore calcimycin stimulate FM1-43 destaining and quantal release in $c s p$ mutants at $32^{\circ} \mathrm{C}$ when depolarization fails to evoke any response. The wild-type-like, calcimycininduced response in csp null mutants indicates that some aspect of the depolarization-dependent calcium signaling pathway must be impaired, either calcium entry, calcium action, or both. Collectively, our results indicate that the csp mutation affects calcium secretion coupling of evoked exocytosis but not vesicle recycling. This supports the hypothesis that CSP links synaptic vesicles to calcium secretion coupling.

Key words: cysteine string protein; CSP; BWSV; calcimycin; synaptic vesicles; neuromuscular junction; exocytosis; endocytosis; vesicle recycling; synaptic transmission
The cysteine string proteins (CSPs) are conserved from invertebrates to mammals, including humans (for review, see Umbach et al., 1995; Buchner and Gundersen, 1997; Zinsmaier, 1997). The various CSP isoforms are associated with diverse vesicle membranes such as granules and synaptic, endocrine, and exocrine vesicles (Mastrogiacomo et al., 1994; Zinsmaier et al., 1994; Braun and Scheller, 1995; Kohan et al., 1995; Chamberlain and Burgoyne, 1996; Chamberlain et al., 1996; van deGoor and Kelly, 1996; Pupier et al., 1997). It has been suggested that the presence of the $\mathrm{J}$ domain in CSP mediates a cooperative interaction with proteins of the heat shock protein 70 family (Cyr et al., 1994). Recently, CSP has been shown to specifically stimulate in vitro the ATPase activity of the clathrin-uncoating ATPase heat shock coguate 70 (HSC70) (Braun et al., 1996; Chamberlain and Burgoyne, 1997) but not the ATPase activity of the $N$ ethylmaleimide-sensitive fusion protein (Braun et al., 1996).

The precise role of CSP is still unknown, although the significance of CSP for neurotransmitter release has been demonstrated by the initial analysis of csp mutant Drosophila strains (Umbach et al., 1994; Zinsmaier et al., 1994). The complete loss of CSP causes a $50 \%$ reduction of evoked neurotransmitter re-

\footnotetext{
Received Sept. 2, 1997; revised Nov. 12, 1997; accepted Nov. 18, 1997.

This work was supported by the Whitehall Foundation, the March of Dimes Birth Defects Foundation, and the National Science Foundation by grants to K.E.Z. We acknowledge the excellent technical support provided by S. Hong.

While this paper was under review, a paper by Umbach and Gundersen (1997) reported a similar stimulation of neurotransmission in $\operatorname{csp}$ mutants with latrotoxin and ionomycin.

Correspondence should be addressed to Dr. Konrad E. Zinsmaier, Department of Neuroscience, University of Pennsylvania School of Medicine, 232a Stemmler Hall, Philadelphia, PA 19104-6074.

Copyright (C) 1998 Society for Neuroscience $\quad 0270-6474 / 98 / 180956-09 \$ 05.00 / 0$
}

lease in mutant flies at $22^{\circ} \mathrm{C}$. With gradually increasing temperatures, evoked release becomes increasingly impaired until it is completely blocked at $>29^{\circ} \mathrm{C}$. This has been demonstrated for adult first-order interneurons of the visual system (Zinsmaier et al., 1994) and for larval neuromuscular junctions (NMJs) (Umbach et al., 1994). However, spontaneous neurotransmitter release persists at high temperatures (Umbach et al., 1994). It has been shown independently that Torpedo CSP seems to act as a positive modulator of $\mathrm{N}$-type calcium channels when coexpressed in frog oocytes (Gundersen and Umbach, 1992). Because CSP is apparently localized to secretory vesicles, this led to the proposal that CSP may link synaptic vesicles to presynaptic calcium channels (Mastrogiacomo et al., 1994). So far, however, no direct molecular interaction has been detected between CSP and presynaptic calcium channels (Martin-Moutot et al., 1996; Pupier et al., 1997).

Our initial electrophysiological studies of csp mutant flies did not determine whether the temperature-sensitive (ts) block of neurotransmitter release is caused by a defect of exocytosis or by a failure of vesicle recycling and genesis that would terminate neurotransmitter release by depleting the vesicle pool of mature synaptic vesicles. Such a potential defect of vesicle recycling, as suggested by Sudhof (1995), seems possible for two reasons. First, the interaction of CSP with HSC70 implies a potential role of CSP in vesicle recycling (Braun et al., 1996; Chamberlain and Burgoyne, 1997), because the only known function of HSC70 at the synaptic terminal is the uncoating of clathrin-coated vesicles (Sudhof, 1995). Second, the ts block of neurotransmitter release in $\operatorname{csp}$ mutants develops slowly with a lag phase of $\sim 2-10 \mathrm{~min}$ (Umbach et al., 1994), which could indicate a depleted vesicle pool. To resolve this issue, we determined whether vesicle recy- 
cling exhibits a ts block in csp mutant larvae that would correlate with the ts block of neurotransmitter release.

\section{MATERIALS AND METHODS}

\section{Drosophila stocks and culture}

Flies were cultured in standard medium at $20^{\circ} \mathrm{C}$. The following Drosophila strains were used: wild-type Berlin, $s h i^{t s I}$ (stock collection, Caltech), $c s p^{E 16}$, and $c s p^{X 1}$. Both $c s p$ mutant alleles represent null mutations (Zinsmaier et al., 1994). The strain shitsl was kept homozygous, whereas the csp alleles were kept heterozygous over the balancer chromosome TM6, Tubby $(T b)$. Absence of the dominant $T b$ marker allowed the selection of $\operatorname{csp}$ homozygous larvae.

\section{Larval body wall muscle preparation}

Climbing third instar larvae were dissected to expose their body wall muscles. For dissection, larvae were placed dorsal side up on a small dish with a thin layer of Sylgard resin in calcium-free HL-3 medium (Stewart et al., 1994). The larvae were pinned down anteriorly and posteriorly and cut along the dorsal midline, and the filleted larvae were pinned out. After removing the viscera, the segmentally repeated larval body wall muscles and the innervating nerve fibers were clearly visible. The muscles were identified as described previously (Johansen et al., 1989). For most of the toxin experiments we removed the CNS to reduce extensive muscle contraction.

\section{FM1-43 assays}

FM1-43 staining induced by $\mathrm{K}^{+}$stimulation. FM1-43 (Molecular Probes, Eugene, OR) staining was induced by $\mathrm{K}^{+}$depolarization essentially as described previously (Ramaswami et al., 1994). However, instead of a saline solution, we used modified HL-3 media (Stewart et al., 1994), which prolonged the survival time of the preparation by several hours. In general, endocytosis was monitored by incubating the larval body wall muscle preparation for $5 \mathrm{~min}$ at the indicated temperature in $60 \mathrm{mM} \mathrm{KCl}$, $4 \mu \mathrm{M}$ FM1-43, and $1.5 \mathrm{mM} \mathrm{CaCl}_{2}$ in HL-k medium (in mM: $15 \mathrm{NaCl}, 20$ $\mathrm{MgCl}_{2}, 10 \mathrm{NaHCO}_{3}, 115$ sucrose, 5 trehalose, and 5 HEPES, pH 7.2). Then the preparation was washed extensively for up to $1 \mathrm{hr}$ at $4^{\circ} \mathrm{C}$ with calcium-free (containing $0.5 \mathrm{~mm}$ EGTA) HL-n medium (in mM: $75 \mathrm{NaCl}$, $20 \mathrm{MgCl}_{2}, 10 \mathrm{NaHCO}_{3}, 115$ sucrose, 5 trehalose, and 5 HEPES, pH 7.2) to remove surface bound FM1-43. For all experiments we used the NMJs of the muscle fibers 6, 7, 12, and 13 as described by Johansen et al. (1989).

FM1-43 destaining induced by $\mathrm{K}^{+}$stimulation. After photodocumentation of FM1-43 staining, the preparation was destained by $\mathrm{K}^{+}$depolarization to monitor depolarization-dependent exocytosis. FM1-43 destaining was stimulated at the indicated temperature for $10 \mathrm{~min}$ with 60 $\mathrm{mm} \mathrm{KCl}$ and $1.5 \mathrm{~mm} \mathrm{CaCl}_{2}$ in $\mathrm{HL}-\mathrm{k}$ medium. For experiments at $32^{\circ} \mathrm{C}$ the larval preparation was preincubated for $15 \mathrm{~min}$ at $32^{\circ} \mathrm{C}$, and all solutions were prewarmed.

FM1-43 staining induced by black widow spider venom stimulation. The larval body wall muscle preparation was incubated at the indicated temperature for $10 \mathrm{~min}$ in $4 \mu \mathrm{M}$ FM1-43 and $1.5 \mathrm{mM} \mathrm{CaCl}_{2}$ in HL-n medium supplemented with 0.6 glands $/ \mathrm{ml}$ crude black widow spider venom (BWSV). Then the preparation was extensively washed with $\mathrm{Ca}^{2+}$-free $\mathrm{Hl}$-n medium at $4^{\circ} \mathrm{C}$ for $1 \mathrm{hr}$.

FM1-43 destaining induced by BWSV stimulation. BWSV induction of FM1-43 destaining was triggered by incubation with 0.6 glands $/ \mathrm{ml}$ BWSV in Hl-n medium. BWSV stimulation was performed as indicated in the presence of external $1.5 \mathrm{mM} \mathrm{CaCl}_{2}$ or in the absence of $\mathrm{Ca}^{2+}(0.5 \mathrm{~mm}$ EGTA).

FM1-43 destaining induced by calcimycin stimulation. For the calcimycin (A23187; Molecular Probes) induction of FM1-43 destaining, we incubated the larval body wall muscle preparation for $10 \mathrm{~min}$ at the indicated temperature in $100 \mu \mathrm{M}$ calcimycin, HL-n medium supplemented with 1.5 mM CaCl 2 or with 0.5 mM EGTA $\left(\mathrm{Ca}^{2+}\right.$-free $)$.

FM1-43 pulse labeling during BWSV stimulation. Larval body wall preparations were equilibrated to $32^{\circ} \mathrm{C}$ for $15 \mathrm{~min}$ in $\mathrm{Hl}-\mathrm{n}$ medium. In the presence of $1.5 \mathrm{mM} \mathrm{CaCl}_{2}$ in $\mathrm{HL}-\mathrm{n}$ medium, exocytosis and endocytosis were stimulated with BWSV $(0.6$ glands $/ \mathrm{ml})$ at $32^{\circ} \mathrm{C}$. Endocytosing vesicles were pulse-labeled by adding $4 \mu \mathrm{M}$ FM1-43 for $5 \mathrm{~min}$. The dye was removed by exchanging the solution twice with fresh BWSV solution (0.6 glands/ml BWSV, $1.5 \mathrm{~mm} \mathrm{CaCl}{ }_{2}, \mathrm{HL}-\mathrm{n}$ medium). The BWSV stimulation was continued for $10 \mathrm{~min}$ at $32^{\circ} \mathrm{C}$. Neurotransmission and BWSV were then inactivated by an incubation with formaldehyde $(2 \%)$ in calcium-free HL-n medium for $5 \mathrm{~min}$, and the preparation was washed extensively for up to $1 \mathrm{hr}$ at $4^{\circ} \mathrm{C}$ in calcium-free $\mathrm{HL}-\mathrm{n}$ medium before viewing. In contrast, in the control experiments the preparations were inactivated immediately after the FM1-43 pulse by the fixative incubation.

FM1-43 imaging. For photomicroscopy the stained preparation was kept in calcium-free HL-n medium, which ensured no spontaneous activity (Ramaswami et al., 1994). FM1-43-stained NMJs were visualized through fluorescein excitation and emission filters with a Reichert Polyvar 2 microscope. Images were acquired by standard microphotography using 400-1600 ASA Ektachrome film and a defined shutter-open time. Images of FM1-43 destaining were obtained either during or after the destaining protocol from the same NMJ that was used earlier for the FM1-43 uptake image. Identical shutter-open times were used for "staining" and "destaining" images. The temperature of the perfusion chamber (RC-20, Warner Instrument Corp.) was monitored and controlled using a stage-mountable heater platform (PH-5, Warner) connected to a TC324A heater controller (Warner).

\section{Preparation of black widow spider venom}

Desiccated and frozen glands from Latrodectus mactans (black widow spider) were purchased from Sigma (St. Louis, MO) or collected by Hatari Invertebrates. The dissected spider glands were homogenized in

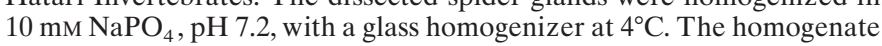
was centrifuged at low speed for $5 \mathrm{~min}$ at $4^{\circ} \mathrm{C}$ to remove debris. We learned that it is essential to remove small molecular weight molecules; otherwise the classic slow-developing BWSV response is mixed with a fast depolarization-dependent induction of transmitter release (instantaneous). The slow response was sensitive to heat treatment, indicating the peptide nature of the agent. Therefore, the spider gland homogenate was gel-filtrated over a Sephadex G-50 spin column at $4^{\circ} \mathrm{C}$ to separate the interfering low molecular weight molecules from the high molecular weight latrotoxin proteins. The eluted proteins were pooled and stored at $-80^{\circ} \mathrm{C}$ in small aliquots. Because this gel filtration only removes small molecules, we still refer to this homogenate as crude BWSV toxin.

\section{Electrophysiology}

All recordings were from muscle fibers 6 and 7 in the anterior abdomen of dissected third instar Drosophila larvae. Intracellular whole-cell recordings of miniature excitatory junction potentials (MEJPs) and evoked excitatory junction potentials (Jan and Jan, 1976) were made with a single microelectrode $(20-40 \mathrm{M} \Omega)$ filled with $3 \mathrm{M} \mathrm{KCl}$. Signals were amplified using an Axopatch-1D amplifier (Axon Instruments) and filtered at $1 \mathrm{kHz}$. The data were digitized at $10 \mathrm{kHz}$ with a Digidata 1200 interface (Axon) and analyzed using the Axoscope application of pCLAMP 6.0 software. To stimulate evoked junction potentials (EJPs), nerve fibers were severed at the base of the ventral ganglion. EJPs were elicited with a suction electrode for $1 \mathrm{msec}$ at one to two times threshold for maximum EJPs. All recordings of spontaneous or toxin-induced release were made in HL-3 medium (Stewart et al., 1994) supplemented with $\mathrm{CaCl}_{2}$ as indicated $\left(0 \mathrm{mM} \mathrm{Ca}{ }^{2+}\right.$ was supplemented with $0.5 \mathrm{~mm}$ EGTA). Calcimycin was added to a final concentration of $50 \mu \mathrm{M}$, tetrodotoxin to $1 \mu \mathrm{g} / \mathrm{ml}$. The temperature of the recording chamber was monitored and controlled using a heater platform, a solution heater (PH-1 or SH-27A, Warner) connected to a TC-324A heater controller (Warner), or both. For the statistical analysis of MEJP frequencies, we determined the number of MEJPs with a minimal amplitude of $0.4 \mathrm{mV}$ over several $15 \mathrm{sec}$ intervals from a continuous recording.

\section{RESULTS}

The ts block of FM1-43 destaining at csp mutant NMJs is consistent with ts block of neurotransmitter release

We used the optical FM1-43 assay developed by Betz and Bewick (1992) to analyze synaptic vesicle endocytosis and exocytosis in csp mutants. This assay facilitates the direct visualization of endocytosis by the activity-dependent vesicular uptake of the lipophilic fluorescent dye FM1-43 (Betz and Bewick, 1992; Betz et al., 1992; Betz and Bewick, 1993). Further stimulation of the synapse causes activity-dependent FM1-43 destaining of the synaptic terminal attributable to exocytosis of dye-loaded synaptic vesicles (for review, see Betz et al., 1996). In Drosophila, this dye has been used successfully to monitor the dynamics of synaptic vesicle endocytosis at the larval NMJ (Ramaswami et al., 1994). 

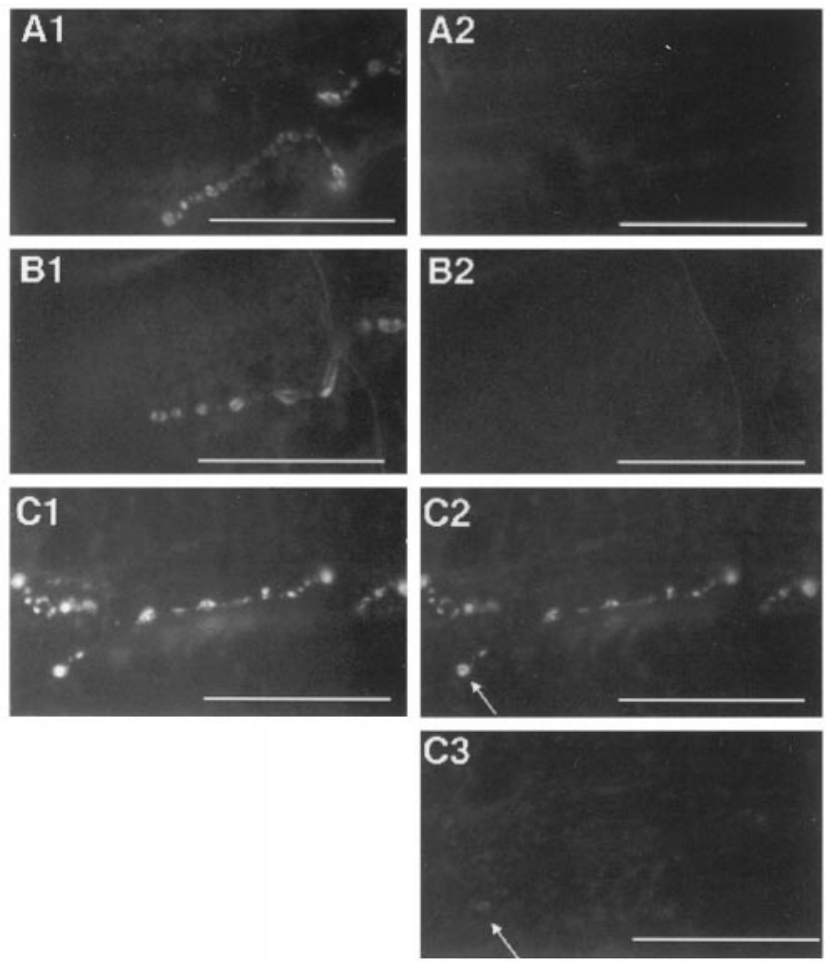

Figure 1. Depolarization fails to trigger FM1-43 destaining in csp mutants at $32^{\circ} \mathrm{C}$. At $22^{\circ} \mathrm{C} \mathrm{K}^{+}$stimulation in the presence of FM1-43 induced a punctate FM1-43 staining of synaptic boutons at all NMJs of wild-type (A1), mutant homozygous $s h i^{t s 1}(B 1)$, and mutant homozygous $\operatorname{csp}^{E 16}$ (C1) muscles. A subsequent $\mathrm{K}^{+}$stimulation at $32^{\circ} \mathrm{C}$ completely destained the FM1-43-labeled synaptic boutons of wild-type $(A 2)$ and $s h i^{t s 1}$ mutant (B2) NMJs. However, $\mathrm{K}^{+}$stimulation at $32^{\circ} \mathrm{C}$ failed to destain the FM1-43-labeled synaptic boutons of $c s p^{E 16}$ mutant NMJs $(C 2)$. After cooling the $c s p^{E 16}$ mutant preparation to $22^{\circ} \mathrm{C}, \mathrm{K}^{+}$depolarization destained the synaptic boutons $(C 3)$ that failed to destain with depolarization at $32^{\circ} \mathrm{C}(C 2)$. Only minute traces of staining could be observed sometimes on large synaptic boutons (arrows). Each series of images $(A-C)$ gives a partial view of the identical NMJ of muscle 6/7 after FM1-43 staining at $22^{\circ} \mathrm{C}(1)$, after FM1-43 destaining at $32^{\circ} \mathrm{C}(2)$, and after FM1-43 destaining at $22^{\circ} \mathrm{C}(3)$. Each series exemplifies at least 10 independent experiments (larvae) for each genotype. Scale bars, $50 \mu \mathrm{m}$.

For the analysis of the ts phenotype of csp null mutations, we chose the temperature of $32^{\circ} \mathrm{C}$ to ensure a complete block of CSP function, because our previous electrophysiological observations demonstrated that the ts block of neurotransmission is complete at $>29^{\circ} \mathrm{C}$ (Umbach et al., 1994). In the following experiments we used shibire (shi) mutant flies as a positive control for a ts block of endocytosis, which slowly depletes the synaptic vesicle pool and terminates neurotransmitter release (Grigliatti et al., 1973; Ikeda et al., 1976; Poodry and Edgar, 1979; Kosaka and Ikeda, 1983a,b).

To test whether evoked synaptic vesicle exocytosis is indeed blocked in csp mutants at a nonpermissive temperature, we stained NMJs of dissected larval body wall preparations with FM1-43 by $\mathrm{K}^{+}$stimulation at $22^{\circ} \mathrm{C}$. After washing, we observed the typical punctate FM1-43 staining on wild-type muscle fibers, reminiscent of synaptic boutons at NMJs (Fig. 1A1). This punctate staining corresponded well with type $1 \mathrm{~b}$ and $1 \mathrm{~s}$ terminals of muscle fibers 6 and 7 (Johansen et al., 1989; Atwood et al., 1993) when viewed by Nomarski optics (data not shown). After confirming the endocytotic uptake of FM1-43, we switched the temperature to $32^{\circ} \mathrm{C}$ to establish the complete ts block of evoked release in csp mutants and stimulated FM1-43 destaining by $\mathrm{K}^{+}$ depolarization. Under these conditions, $\mathrm{K}^{+}$stimulation consistently failed to destain FM1-43 labeled synaptic boutons of $c s p$ mutant NMJs (Fig. 1C2). To demonstrate that this ts block of FM1-43 destaining is reversible, we cooled the preparation to $22^{\circ} \mathrm{C}$, at which the FM1-43 labeled boutons of csp mutants were destained completely upon depolarization (Fig. 1C3). In control preparations the high temperature had no effect on the destaining of FM1-43-labeled synaptic boutons in wild-type or shi mutant larvae that were completely destained on $\mathrm{K}^{+}$depolarization at $32^{\circ} \mathrm{C}$ (Fig. 1A2,B2). The comparison of the csp and shi mutant phenotypes suggests that different mechanisms of vesicle trafficking are defective in each mutant. The ts block of depolarizationdependent FM1-43 destaining implies that synaptic vesicles are present in csp mutant terminals but unable to respond to nerve stimulation.

\section{Black widow spider venom bypasses the ts block of neurotransmitter release in csp mutants}

To characterize CSP function further, we tested whether the action of BWSV is impaired in csp mutants at $32^{\circ} \mathrm{C}$ when evoked release is blocked. Crude BWSV or its purified components, the latrotoxins, have been shown to induce a massive increase in the frequency of quantal transmitter release at vertebrate and invertebrate nerve endings, including Drosophila (Clark et al., 1970; Magazanik et al., 1992; Ramaswami et al., 1994; Storchak et al., 1994; Broadie et al., 1995; Linial et al., 1995). After staining NMJs with FM1-43 at $22^{\circ} \mathrm{C}$, we switched the temperature to $32^{\circ} \mathrm{C}$ and tested whether ts block of evoked FM1-43 destaining had been established. No significant amount of FM1-43 staining was released from the boutons of $c s p$ mutants upon $\mathrm{K}^{+}$stimulation (Fig. 2A1). However, a subsequent stimulation with BWSV induced a complete destaining of FM1-43 at csp mutant NMJs in the presence of $\mathrm{Ca}^{2+}$ (Fig. 2A2). FM1-43 destaining could also be triggered by BWSV in the absence of external $\mathrm{Ca}^{2+}$; however, it required up to 60 min to destain $c s p$ or wild-type NMJs (data not shown). These features are consistent with earlier reports demonstrating BWSV-induced release in the presence and absence of extracellular calcium (Clark et al., 1970; Magazanik et al., 1992; Ramaswami et al., 1994; Storchak et al., 1994; Broadie et al., 1995; Linial et al., 1995; Barnett et al., 1996). Independent recordings of MEJPs at $31^{\circ} \mathrm{C}$ consistently showed a dramatic increase in the frequency of quantal release after BWSV application, which was similar in wild-type and $\operatorname{csp}^{X 1}$ mutant larvae (Fig. 2B-C). Several minutes after the application of BWSV, the basal MEJP frequency of $3.7 \pm 0.2 \mathrm{~Hz}($ mean $\pm \mathrm{SEM} ; n=3)$ for wild type and $3.7 \pm 0.4 \mathrm{~Hz}($ mean $\pm \mathrm{SEM} ; n=3)$ for $c s p^{X 1}$ increased within $30 \mathrm{sec}$ to a peak frequency of $61 \pm 6 \mathrm{~Hz}$ (mean \pm $\mathrm{SEM} ; n=3)$ for wild type and $70 \pm 5 \mathrm{~Hz}($ mean $\pm \mathrm{SEM} ; n=3)$ for $\operatorname{csp} p^{X 1}$ before it steadily declined to the basal level. There was no significant difference between the peak frequency of BWSVinduced release for wild type and $\operatorname{csp}$ mutants ( $p=0.27$, Student's $t$ test).

\section{Calcimycin triggers calcium-dependent release at csp mutant $\mathrm{NMJ}$ at $32^{\circ} \mathrm{C}$ at a time when evoked release is blocked}

Because the wild-type-like BWSV response in csp mutant larvae indicated that the mutation does not affect aspects of quantal release, we speculated that the $\operatorname{csp}$ mutation may indeed interrupt calcium signaling with depolarization at $32^{\circ} \mathrm{C}$. Our previous work demonstrated that the csp mutation interferes with the calcium sensitivity of evoked release at $22^{\circ} \mathrm{C}$, suggesting that CSP is involved in calcium secretion coupling (Umbach et al., 1994). 


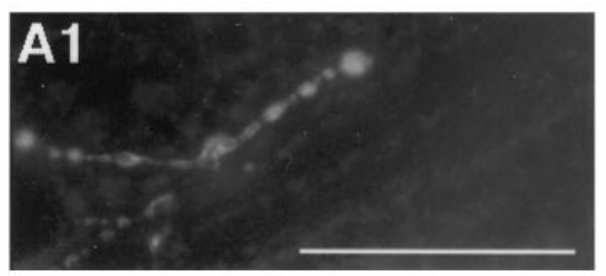

\section{B}
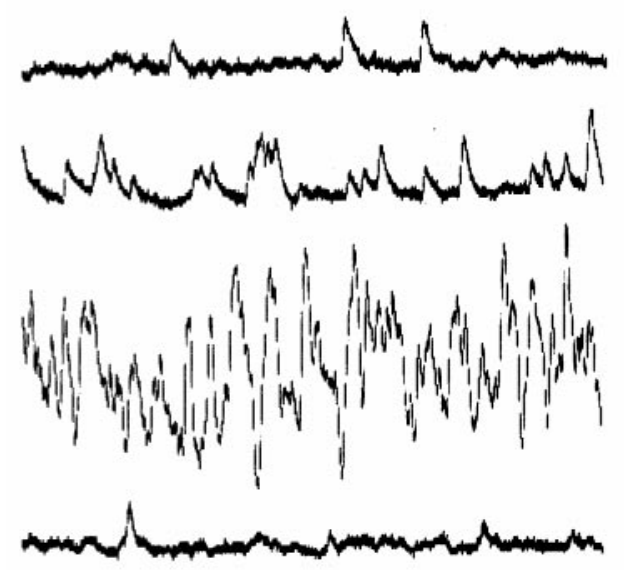

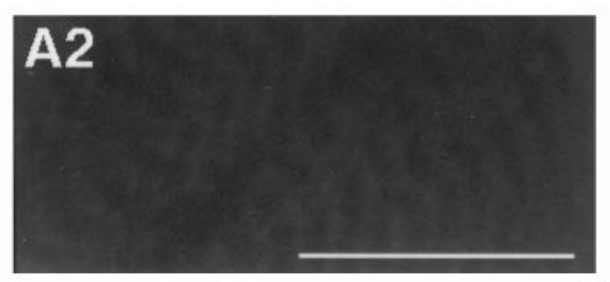

C
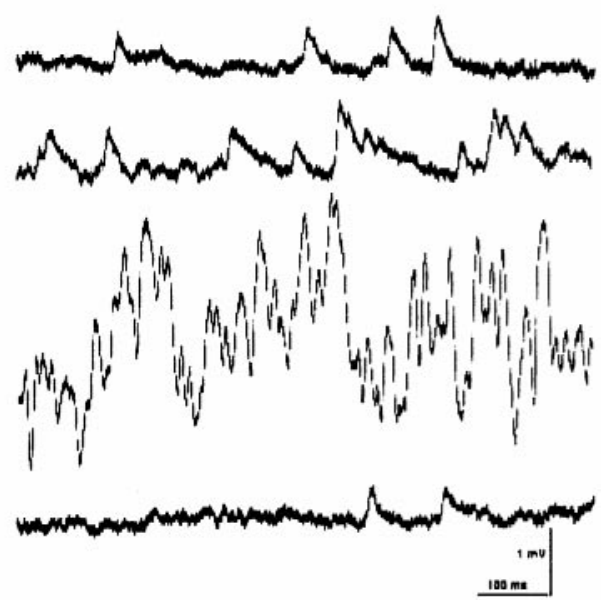

Figure 2. BWSV stimulates exocytosis in $\operatorname{csp}$ mutant NMJs at $32^{\circ} \mathrm{C}$. $A$, Larval NMJs of muscle 6/7 from homozygous $c s p^{E 16}$ larvae were labeled by FM1-43 $\mathrm{K}^{+}$-induced depolarization at $22^{\circ} \mathrm{C}$ (data not shown). The ts block of $\mathrm{K}^{+}$-induced FM1-43 destaining at $32^{\circ} \mathrm{C}$ was confirmed, and no significant amounts of FM1-43 stain were released $(A 1)$. Subsequent FM1-43 destaining was stimulated with BWSV in the presence of external $\mathrm{Ca}^{2+}$ at $32^{\circ} \mathrm{C}$. This completely destained the $c s p^{E 16}$ synaptic boutons of the same NMJ (A2) that failed to destain with the previous $\mathrm{K}^{+}$depolarization $(A 1)$. The images represent eight independent experiments (larvae). $B, C, \mathrm{Re}-$ cordings of BWSV-induced quantal release from wild-type NMJs $(B)$ and $c s p^{X 1}$ homozygous NMJs $(C)$ at $31^{\circ} \mathrm{C}$. MEJPs were recorded continuously from muscle fiber 6 in the presence of external $\mathrm{Ca}^{2+}$ at $31^{\circ} \mathrm{C}$. The first trace $(B, C)$ shows a typical example of MEJPs during continuous recording before the application of BWSV. After the application of BWSV $(15-180 \mathrm{sec})$ a rapid increase of up to $50 \mathrm{~Hz}$ in MEJP frequency was observed within a $30 \mathrm{sec}$ interval (trace $2, B, C$ ). The peak frequencies (highest 15 sec interval observed per preparation) of this irregular response were $71 \mathrm{~Hz}$ for wild type and $77 \mathrm{~Hz}$ for $c s p^{X 1}$ mutants and lasted for $\sim 1-2$ min (trace 3, B, C). Within the next minute the MEJP frequency of the BWSV response declined steadily to its original level (trace 4, B, C). After this rather silent "depletion phase," the BWSV response recovered and kept oscillating repeatedly, but with a significantly lower peak frequency (data not shown). With the onset of the high-frequency release induced by BWSV, a dramatic depolarization of the muscle was observed. The muscle potentials for these particular recordings dropped from $-65 \mathrm{mV}$ to $-35 \mathrm{mV}$ (wild type, $B$ ) and from $-70 \mathrm{mV}$ to -50 $\mathrm{mV}\left(\operatorname{csp} p^{X 1}, C\right)$. Both potentials recovered as soon as the frequency of release declined to its original value. Only recordings that recovered to the original muscle potential during the depletion phase were used for the analysis. Scale bars, $50 \mu \mathrm{m}$.

However, because a morphological change of the NMJ could not be ruled out as an alternative cause for this phenotype, we gathered further evidence for this speculation. Calcium ionophores, such as calcimycin and ionomycin, have been shown to bypass voltage-gated calcium channels, to elevate intracellular calcium concentrations, and to trigger neurotransmitter exocytosis in synaptosomes (Verhage et al., 1991; Alder et al., 1992) and in hippocampal or bipolar cell synaptic terminals (von Gersdorff and Matthews, 1994; Capogna et al., 1996). However, calcium ionophores appear less effective than calcium channels in activating exocytosis (Verhage et al., 1991; von Gersdorff and Matthews, 1994; Capogna et al., 1996).

To test whether the calcium signaling pathway is affected by the $\operatorname{csp}$ mutation at $32^{\circ} \mathrm{C}$, we tested calcimycin (A-23187) for its ability to trigger exocytosis in $\operatorname{csp}$ mutants. After $\mathrm{K}^{+}$depolarization failed to induce FM1-43 destaining at $32^{\circ} \mathrm{C}$ (Fig. $3 A 1$ ), we stimulated the preparation with calcimycin in the absence of external $\mathrm{Ca}^{2+}$ at $32^{\circ} \mathrm{C}$, which induced no significant destaining the synaptic boutons (Fig. $3 A 2$ ). However, subsequent incubation at $32^{\circ} \mathrm{C}$ with calcimycin and external $\mathrm{Ca}^{2+}$ induced a complete destaining of the FM1-43-labeled csp mutant synaptic boutons (Fig. 3A3). The calcimycin-induced FM1-43 destaining in the presence of external calcium could be blocked by preincubation of the preparation with BAPTA-AM ester (Fig. 3B2). Electrophysiological recordings from NMJs confirmed the ability of calcimycin to increase the frequency of quantal release significantly in wild type and in $\operatorname{csp}$ mutants at $32^{\circ} \mathrm{C}$ (Fig. $3 C, D$ ). The frequency of quantal release increased from the basal spontaneous level of $3.5 \pm 1.4 \mathrm{~Hz}$ $($ mean $\pm \operatorname{SEM} ; n=3$ ) for wild type and $2.9 \pm 1.2 \mathrm{~Hz}$ (mean \pm
SEM; $n=3$ ) for $c s p^{X 1}$ to a mean peak frequency of $42 \pm 3 \mathrm{~Hz}$ $($ mean $\pm \mathrm{SEM} ; n=3$ ) for wild type and $41 \pm 5 \mathrm{~Hz}$ (mean \pm SEM; $n=3$ ) for csp mutants. Because there is no significant difference between the calcimycin-induced quantal release in $c s p$ mutants and the calcimycin response in wild type $(p=0.92$, Student's $t$ test), this suggests that there is no effect of the csp mutation on the calcimycin response.

\section{csp mutant flies exhibit no ts defect of vesicle recycling that would correlate with the complete ts block of neurotransmitter release}

The wild-type-like BWSV-mediated increase of quantal release in $\operatorname{csp}$ mutants (Fig. $2 B, C$ ) provided some indirect evidence that the synaptic vesicle pool at larval NMJs is not affected by the $c s p$ mutation. To gather direct evidence for this speculation, we assayed evoked endocytosis at $32^{\circ} \mathrm{C}$ in $\operatorname{csp}$ mutants. At this temperature, we never observed the typical punctate FM1-43 staining of synaptic boutons at $c s p$ or shi mutant synaptic boutons on $\mathrm{K}^{+}$ stimulation (Fig. 4A1,B1). However, wild-type muscle fibers consistently showed endocytotic uptake of FM1-43 (data not shown). Because both shi and csp phenotypes are temperature-sensitive, we cooled the same preparations to $22^{\circ} \mathrm{C}$ and repeated the FM143-staining protocol. This stained csp and shi mutant NMJs with FM1-43 (Fig. 4A2,B2). The ts block of FM1-43 uptake in the shi control flies at $32^{\circ} \mathrm{C}$ is consistent with similar observations of FM1-43 uptake (Ramaswami et al., 1994). The lack of FM1-43 uptake in csp mutant NMJs normally would be suggestive of a ts defect of endocytosis. However, because evoked exocytosis is blocked in $\operatorname{csp}$ mutants at $32^{\circ} \mathrm{C}$ (Fig. $1 C 2$ ), but not in shi mutant 
Figure 3. Calcimycin induces FM1-43 destaining and elevated quantal release in csp mutants at $>30^{\circ} \mathrm{C}$. $A$, NMJs of homozygous $\operatorname{csp}^{E 16}$ mutant larvae were stained with FM1-43 by $\mathrm{K}^{+}$depolarization at $22^{\circ} \mathrm{C}$ (data not shown), and the ts block of FM1-43 destaining was confirmed. At $32^{\circ} \mathrm{C} \mathrm{K}^{+}$stimulation failed to destain the labeled synaptic boutons (A1). Calcimycin (A23187) stimulation of FM1-43 destaining in calcium-free medium at $32^{\circ} \mathrm{C}$ released little, if any, FM1-43 dye from the synaptic boutons (A2). However, calcimycin stimulation in the presence of external $\mathrm{Ca}^{2+}$ at $32^{\circ} \mathrm{C}$ destained the same NMJ (A3) that failed to destain with the previous two stimuli $(A 1, A 2)$. The images represent five independent preparations. $B$, After FM1-43 staining at $22^{\circ} \mathrm{C}$ (data not shown) and confirming the ts block of evoked FM1-43 destaining at $32^{\circ} \mathrm{C}$ (B1), NMJs of homozygous $\operatorname{csp}{ }^{E 16}$ mutants were treated for $5 \mathrm{~min}$ with $10 \mu \mathrm{M}$ BAPTA-AM ester in calcium-free medium. After removal of the residual external BAPTA ester, subsequent calcimycin stimulation failed to destain the FM1-43labeled and BAPTA-loaded synaptic boutons at $32^{\circ} \mathrm{C}(B 2)$. The images represent five preparations. $C, D$, MEJPs were recorded contin-uously at $31^{\circ} \mathrm{C}$ in the presence of $0.5 \mathrm{mM} \mathrm{Ca}^{2+}$ and $1 \mu \mathrm{g} / \mathrm{ml}$ TTX from muscle fiber 6 of wild-type $(C)$ and $\operatorname{csp}^{X 1}$ mutant $(D)$ larvae. The first trace $(C, D)$ represents MEJP recordings before the application of calcimycin. Trace 2 shows the onset of the response $(C, D)$ shortly after the application (30 sec) of calcimycin. Trace 3 shows the highest activity, which was on average $42 \pm 3 \mathrm{~Hz}\left(\right.$ mean $\pm \mathrm{SEM} ; n=3$ ) for wild-type and $41 \pm 5 \mathrm{~Hz}(\mathrm{mean} \pm \mathrm{SEM} ; n=3)$ for $c s p^{X 1}$ mutant larvae recorded before fast muscle depolarizations hindered any further electrophysiological analysis. Muscle potentials were $-67 \mathrm{mV}$ for wild-type $(C)$ and $-57 \mathrm{mV}$ for $c s p^{X 1}$ mutant $(D)$ larvae. Scale bars, $50 \mu \mathrm{m}$.
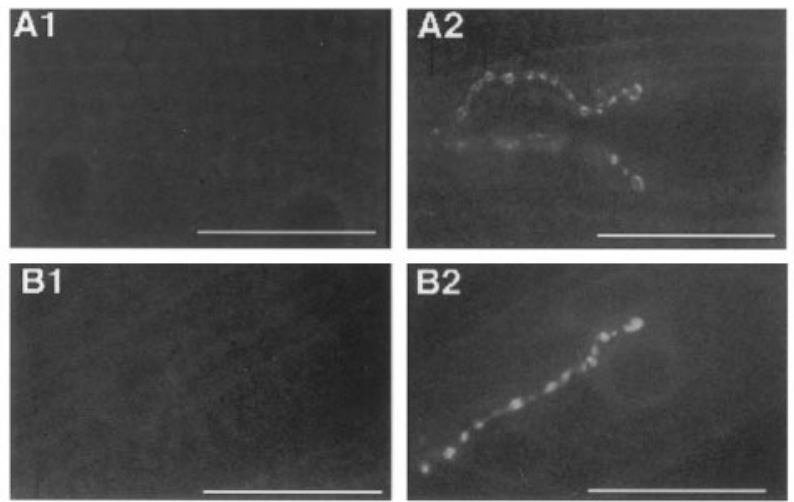
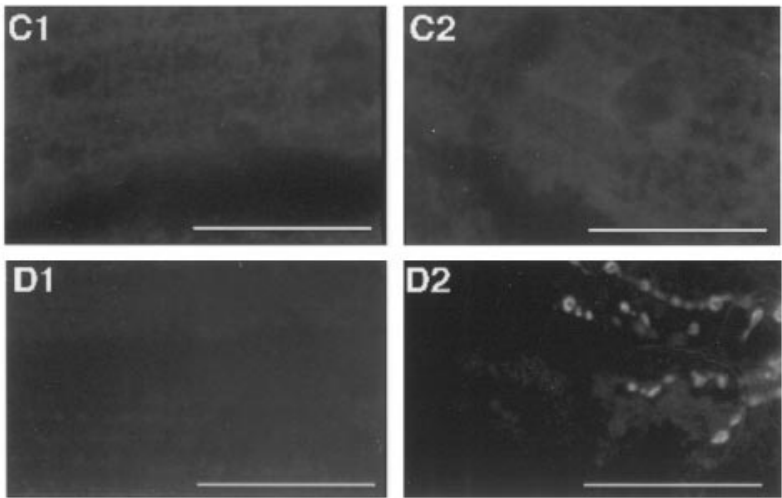

Figure 4. Depolarization-induced, but not BWSV-induced, FM1-43 staining is blocked at $\operatorname{csp}$ mutant NMJs at $32^{\circ} \mathrm{C}$. At $32^{\circ} \mathrm{C} \mathrm{K}{ }^{+}$depolarization failed to induce a significant FM1-43 uptake in synaptic boutons of homozygous $s h i^{t s I}$ NMJs $(A 1)$ and homozygous $c s p^{E 16}$ NMJs $(B 1)$. After cooling to $22^{\circ} \mathrm{C}$, $\mathrm{K}^{+}$stimulation stained synaptic boutons of $s h i^{t s 1}$ NMJs (A2) with FM1-43 and $c s p^{E 16}$ NMJs $(B 2)$. For the next series of experiments $(C, D)$, the mutant ts block of FM1-43 uptake at $32^{\circ} \mathrm{C}$ was confirmed with $\mathrm{K}^{+}$depolarization for homozygous shits ${ }^{t s}$ NMJ $(C 1)$ and homozygous $c s p^{E 16} \mathrm{NMJs}(D 1)$. No staining was observed in either case. The subsequent BWSV stimulation in the presence of external $\mathrm{Ca}^{2+}$ at $32^{\circ} \mathrm{C}$ failed to induce any uptake of $\mathrm{FM} 1-43$ dye at $s h i^{t s I}$ mutant synaptic boutons $(C 2)$. However, BWSV stimulation at $32^{\circ} \mathrm{C}$ induced a strong uptake of FM1-43 dye at $c s p^{E 16}$ mutant NMJs $(D 2)$. Each series of images represents at least five independent experiments and shows partial NMJs of muscle 6/7 except in $D$. Scale bars, $50 \mu \mathrm{m}$.

flies (Fig. 1B2), we considered an alternative explanation that the observed ts block of evoked endocytosis may be secondary and attributable to a ts block of evoked exocytosis. In this case, the stimulation of exocytosis by $\mathrm{K}^{+}$depolarization simply would not generate any membranes to be recycled. To test this alternative explanation, we stimulated endocytosis with BWSV and observed FM1-43 dye uptake at $c s p$ mutant boutons at $32^{\circ} \mathrm{C}$ (Fig. $4 D 2$ ). In contrast, we never detected any FM1-43 uptake in shi mutant terminals with BWSV stimulation at $32^{\circ} \mathrm{C}$ (Fig. 4C2). This indicates that the ts block of $\mathrm{K}^{+}$-induced FM1-43 uptake in csp mutants at $32^{\circ} \mathrm{C}$ is caused by a defect of depolarizationdependent exocytosis. However, an alternative possibility may be that BWSV activates a parallel route of endocytosis, which is blocked in shi but not in csp mutant flies.

An interesting general feature of BWSV-poisoned nerve terminals in the absence of external $\mathrm{Ca}^{2+}$ is that they quickly become depleted of synaptic vesicles, because the endocytosis of BWSV-secreted vesicles is blocked (Ceccarelli et al., 1979; Fritz et al., 1980). These electron microscopic studies correlate well with the minute quantity of FM1-43 dye uptake in the absence of 


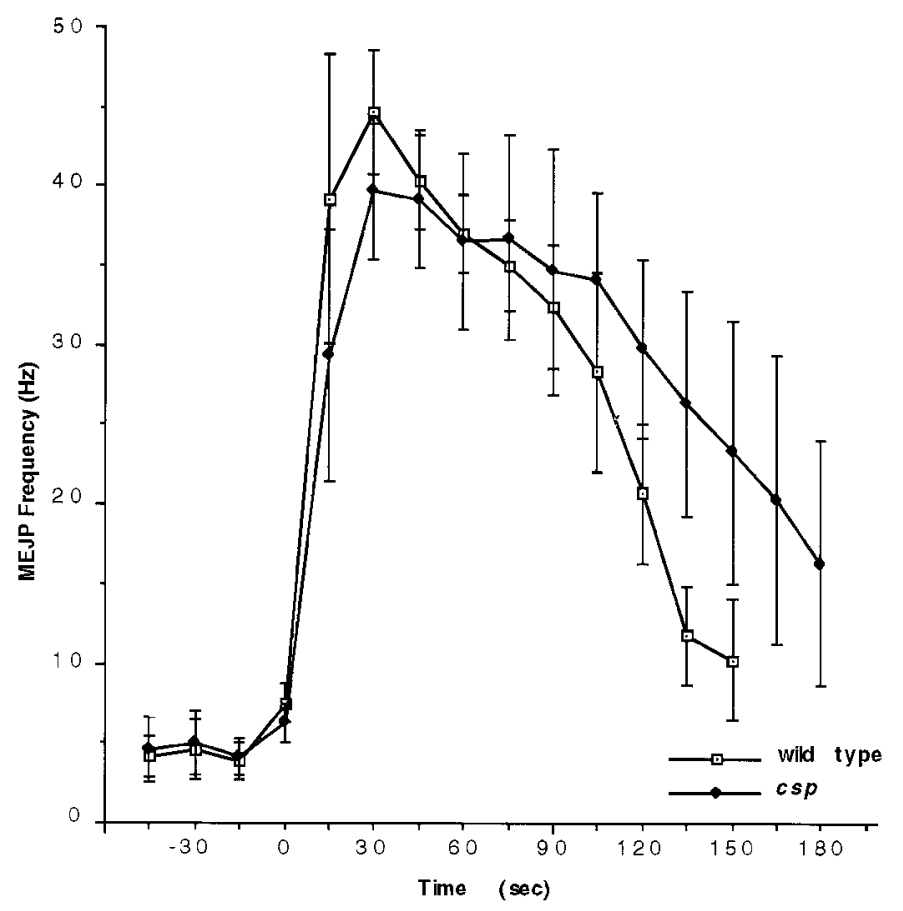

Figure 5. The BWSV-induced increase in quantal release of csp mutants appears similar in frequency and duration to wild type when endocytosis is blocked. MEJPs were recorded continuously from muscle fiber 6 in the absence of calcium at $31^{\circ} \mathrm{C}$. We compared the BWSV-induced increase of the MEJP frequency for wild-type and for $c s p^{X 1}$ mutant larvae. The MEJP frequency was determined in $15 \mathrm{sec}$ intervals. Because the onset of the toxin response differed significantly from trial to trial, we adjusted the curves for the onset of the BWSV response, which was identified as the first interval showing approximately twice the spontaneous frequency observed before drug application $[4.2 \pm 1.5 \mathrm{~Hz}$ (mean $\pm \mathrm{SEM} ; n=3)$ for wild type and $4.6 \pm 1.6 \mathrm{~Hz}$ (mean $\pm \mathrm{SEM} ; n=3$ ) for $\left.c s p^{X 1}\right]$. The responses of three independent recordings (3 larvae for each genotype) were averaged and plotted. The curves appear similar for their peak frequencies, the decline in MEJP frequency, and the duration of the response.

$\mathrm{Ca}^{2+}$ (Ramaswami et al., 1994; Henkel and Betz, 1995). The ability to stimulate exocytosis and to block endocytosis simultaneously allows for, in principle, an electrophysiological estimate of the amount of the BWSV-releasable synaptic vesicle pool. Thus, we compared the frequency of BWSV-induced quantal release in the absence of $\mathrm{Ca}^{2+}$ in wild type and csp mutants. We found that the mean MEJP frequencies and the time course of the BWSV-induced response in $\operatorname{csp}$ mutants at $32^{\circ} \mathrm{C}$ are similar to those of wild type at $32^{\circ} \mathrm{C}$ (Fig. 5). Although the BWSV responses are rather variable, even in wild type, there is almost certainly no correlation of the BWSV response with the ts block of evoked release in csp mutants. The wild-type-like features of BWSVinduced quantal release imply that the pool of BWSV-releasable vesicles in $\operatorname{csp}$ mutants is similar to that of wild type at $32^{\circ} \mathrm{C}$.

To gather further evidence for a potential ts defect in a late step of vesicle recycling, such as the uncoating of clathrin-coated vesicles, we tested the "functionality" of vesicles by asking whether csp mutant vesicles were able to exocytose if they were recycled at $32^{\circ} \mathrm{C}$. To address this question experimentally, we used an FM1-43 pulse-chase protocol and stimulated exocytosis and endocytosis with BWSV. We pulsed the preparation with FM1-43 dye at $32^{\circ} \mathrm{C}$ to label newly endocytosed vesicles. After the dye had been omitted, the BWSV stimulation was continued at $32^{\circ} \mathrm{C}$ to trigger the release of the stained vesicles. We always

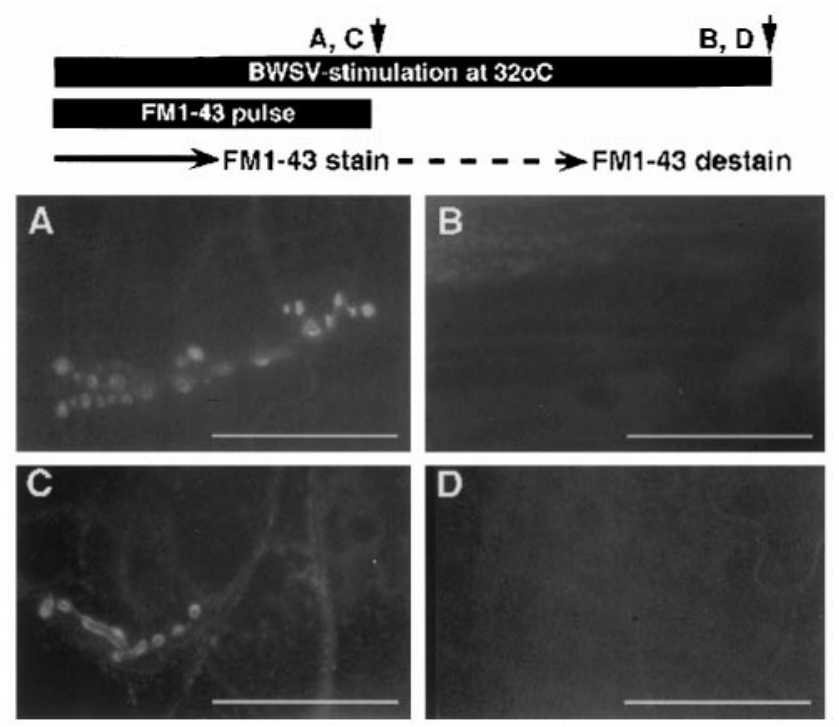

Figure 6. FM1-43 dye endocytosed at $32^{\circ} \mathrm{C}$ by BWSV stimulation can be released immediately at $32^{\circ} \mathrm{C}$ at $c s p$ mutant NMJs. At $32^{\circ} \mathrm{C}$, exocytosis and subsequent endocytosis were induced with BWSV in the presence of external $\mathrm{Ca}^{2+}$. To label freshly endocytosed vesicles (FM1-43 staining), the preparation was pulsed with FM1-43 dye. After omitting the dye the BWSV stimulation was continued at $32^{\circ} \mathrm{C}$ (FM1-43 destaining). Using this protocol, neither wild-type $(B)$ nor $c s p^{E 16}$ mutant $(D)$ NMJs were stained with FM1-43 dye. However, control experiments, in which BWSV and neurotransmission were inactivated after the FM1-43 pulse, always showed FM1-43-stained synaptic boutons as exemplified for wild type $(A)$ and $c s p^{E 16}$ mutants $(C)$. The images represent six independent trials using three larvae for each genotype per experiment $(A-D)$.

observed FM1-43 destaining of csp mutant or wild-type synaptic boutons with this protocol (Fig. 6B,D). Because the controls studied in parallel always generated FM1-43-stained NMJs in wild type (Fig. 6A) and in csp mutants (Fig. $6 C$ ), we assume that the unstained synaptic boutons (Fig. $6 B, D$ ) were stained during the FM1-43 pulse and became destained after the dye had been omitted. Thus, FM1-43 stain that has been taken up at $32^{\circ} \mathrm{C}$ with BWSV stimulation can be released properly at $32^{\circ} \mathrm{C}$ in wild type and in csp mutants. This suggests that synaptic vesicles of $c s p$ mutants that were endocytosed at $32^{\circ} \mathrm{C}$ are able to exocytose at $32^{\circ} \mathrm{C}$ with BWSV stimulation.

\section{DISCUSSION}

Two alternative models of CSP function have been suggested. The first suggested that CSP may link synaptic vesicles to presynaptic calcium channels and thereby may modulate neurotransmission (Mastrogiacomo et al., 1994). This hypothesis was supported primarily by the positive modulation of N-type calcium channels when Torpedo CSP cDNA was coexpressed in frog oocytes (Gundersen and Umbach, 1992). Our previous phenotypic analysis of csp null mutations in Drosophila revealed a ts block of neurotransmission at $>29^{\circ} \mathrm{C}$ (Umbach et al., 1994; Zinsmaier et al., 1994). This observation and the reduced calcium sensitivity of evoked release at $22^{\circ} \mathrm{C}$ (Umbach et al., 1994) were consistent with the "channel hypothesis." Alternatively, it has been suggested that CSP may function at a step in the clathrin-dependent recycling of synaptic vesicles by cooperating with the clathrin-uncoating ATPase HSC70 (Sudhof, 1995). The slowly developing ts phenotype of csp mutant flies (Umbach et al., 1994) and the discovery that mammalian CSP interacts in vitro with HSC70 (Braun et al., 1996; 
Chamberlain and Burgoyne, 1997) strengthened the "recycling hypothesis."

Two assumptions were critical for our analysis of a potential vesicle-recycling phenotype. First, a potential genetic defect in recycling should be most severe at $>30^{\circ} \mathrm{C}$ and should correlate with the complete ts block of evoked neurotransmitter release in csp deletion mutants. Second, a ts-recycling defect should deplete the releasable synaptic vesicle pool in csp mutants, as seen in the shi mutation. Therefore, we compared the dynamics of synaptic vesicle exocytosis and endocytosis of csp mutants with those of $s h i$ mutant flies using the optical FM1-43 assay developed by Betz and Bewick (1992). First, we tested whether evoked exocytosis, endocytosis, or both are blocked in $\operatorname{csp}$ mutants at $32^{\circ} \mathrm{C}$. We demonstrated that at $32^{\circ} \mathrm{C}$, FM1-43 destaining with $\mathrm{K}^{+}$stimulation is blocked at $\operatorname{csp}$ but not at shi mutant synaptic boutons (Fig. 1). The persistence of FM1-43 staining at csp mutant boutons indicates a primary block of evoked exocytosis at $32^{\circ} \mathrm{C}$ and argues against the theory of a depleted vesicle pool. However, when we monitored endocytosis, we observed that at $32^{\circ} \mathrm{C}$ both $c s p$ and $s h i$ mutant NMJs exhibited a depolarization-dependent block of FM1-43 dye uptake (Fig. 4). Because evoked exocytosis is blocked in csp mutant larvae, but not in shi mutant larvae (Fig. 1), we considered the possibility that the depolarization-dependent block of endocytosis may be secondary and may be caused by the primary block of evoked exocytosis. This possibility is consistent with the observation that endocytotic activity is coupled tightly to exocytosis (Heuser and Reese, 1973; Betz and Wu, 1995; Ryan and Smith, 1995). We tested our speculation by stimulating endocytosis with BWSV, the exocytotic action of which is not impaired by the $\operatorname{csp}$ mutation (Fig. 3). The BWSV stimulation labeled synaptic boutons of $\operatorname{csp}$ mutant $\mathrm{NMJs}$ at $32^{\circ} \mathrm{C}$ but not of shi mutant NMJs, which exhibited a complete block of dye uptake (Fig. 4). A similar result, FM1-43 staining in csp and no staining in shi mutants, has been obtained when endocytosis was triggered with calcimycin (data not shown), which makes it less likely that BWSV activates a parallel pathway of endocytosis that does not require CSP function. The possibility of a maverick route of endocytosis stimulated by BWSV is also diminished by the result that FM1-43 staining with BWSV stimulation is readily releasable (Fig. 6). Therefore, our results provide compelling evidence that the ts block of evoked endocytosis is a secondary effect of the primary ts block of depolarization-dependent exocytosis in csp mutants.

To exclude the possibility of a severe ts defect in csp mutants during late steps of vesicle recycling, we estimated the size of the BWSV-releasable vesicle pool. This was possible because BWSV triggers vesicle fusion in the absence of calcium when endocytosis is blocked (Ceccarelli et al., 1979; Fritz et al., 1980; Ramaswami et al., 1994; Henkel and Betz, 1995). As we show in Figure 5, the $\mathrm{Ca}^{2+}$-independent BWSV response of quantal release in wildtype larvae is similar to that of csp mutant larvae in its time course and frequency. This is inconsistent with the speculation that the $\operatorname{csp}$ mutation may cause a ts block of vesicle recycling and may deplete the vesicle pool. In addition, we tested the functionality of $c s p$ mutant vesicles that were recycled at $32^{\circ} \mathrm{C}$. Here, we assume that immature vesicles cannot exocytose, because most steps of vesicle genesis, such as clathrin uncoating, are thought to be essential for vesicles to exocytose. We pulse-labeled synaptic vesicles with FM1-43 as they were endocytosed at $32^{\circ} \mathrm{C}$ and tested whether these vesicles were immediately able to exocytose. If the CSP protein is required for vesicle genesis, theoretically we should observe a significant deficit of FM1-43 destaining in this experiment. We found no evidence of any ts impairment of FM1-43 destaining in csp mutants in this experiment (Fig. 6), suggesting that essential steps of vesicle genesis are not affected by the $t s$-csp mutation. However, a less penetrant non-ts defect of vesicle recycling cannot be ruled out. The experiment also indicates that BWSV stimulates a normal route of endocytosis, leading to readily releasable vesicles, which supports the physiological significance of our results. In summary, our analysis of vesicle recycling shows that the csp mutation does not cause a ts block of synaptic vesicle recycling that would correlate with the ts block of evoked neurotransmission.

Consistent with the assumption that the csp mutation blocks evoked exocytosis, we demonstrated that depolarizationdependent FM1-43 destaining of csp mutant synaptic boutons is blocked reversibly at $32^{\circ} \mathrm{C}$ (Fig. 1), which correlates well with earlier recordings of the ts block of evoked release (Umbach et al., 1994). Interestingly, we discovered that the csp mutation does not affect the stimulation of neurotransmitter release induced by BWSV or calcimycin (Figs. 2, 3). Similar results with a purified component of BWSV, $\alpha$-latrotoxin, have been obtained for $\operatorname{csp}$ mutant larvae (Umbach et al., 1995). The ability of BWSV to induce quantal release and FM1-43 destaining in csp mutants at nonpermissive temperatures (Fig. 2) implies that the CSPdependent step of evoked release must be upstream of the quantal release step stimulated by BWSV. This is interesting because the BWSV response is inhibited in synaptobrevin and syntaxin mutant embryos of Drosophila (Broadie et al., 1995). There, the failure of BWSV to induce quantal release, together with the observed docking of synaptic vesicles, led to the conclusion that synaptobrevin and syntaxin proteins function downstream of vesicle docking (Broadie et al., 1995). Consequently, the action of BWSV in $c s p$ mutants argues that CSP is required upstream of synaptobrevin and syntaxin function.

All of our results are consistent with the conclusion that the csp mutation may interfere with evoked exocytosis. To obtain further evidence, we tested the calcium ionophore calcimycin to trigger release in $\operatorname{csp}$ mutant larvae at $32^{\circ} \mathrm{C}$ when the ts block of evoked release is most severe. As speculated, calcimycin requires extracellular calcium to induce the destaining of FM1-43-labeled synaptic boutons in csp mutants that failed to destain with depolarization at $32^{\circ} \mathrm{C}$ (Fig. $3 A 1-3$ ). These FM1-43 results are supported strongly by recordings from mutant NMJs that demonstrate the ability of calcimycin to increase quantal release in csp mutants at $32^{\circ} \mathrm{C}$ as in wild type (Fig. $3 C-D$ ). Because calcium ionophores trigger neurotransmitter release by bypassing voltage-gated calcium channels (Alder et al., 1992; Capogna et al., 1996), the calcimycin stimulation of exocytosis in $\operatorname{csp}$ mutant larvae at $32^{\circ} \mathrm{C}$ suggests that the ts mutant defect must be upstream of, or within, the calcium signaling cascade mediating evoked exocytosis. Because we demonstrated previously that the propagation of action potentials is normal in csp mutants (Umbach et al., 1994), we conclude that the depolarization-dependent calcium signaling pathway of evoked release is mutated. Thus, either calcium entry (by voltage-gated calcium channels), its release-triggering activity (calcium receptors and downstream signaling pathway), or both mechanisms must be defective in csp mutants. This conclusion is consistent with the recent observation that the time constant of the evoked current decay is increased in csp null mutations at $16-18^{\circ} \mathrm{C}$, implying that CSP helps to synchronize evoked release (Heckmann et al., 1997).

Our electrophysiological recordings of the calcimycin response in $c s p$ mutants are not able to differentiate conclusively between 
defects of calcium entry and calcium action. However, they point toward a defect of calcium entry, because a defect of calcium action should reduce significantly, if not abolish, the calcimycin response for a given calcimycin or calcium concentration. Because this has not been observed (Fig. 3), CSP may be required to modulate the kinetics of calcium entry, as has been proposed previously (Mastrogiacomo et al., 1994). Alternatively, in cooperation with HSC70, the CSP protein may cluster calcium entry sites in close proximity to neurotransmitter release sites. The significance of clustering calcium channels with vesicle release sites has been described by the single calcium domain model (Stanley, 1993) and is supported by recent findings that severing the physical interaction between presynaptic calcium channels and several synaptic proteins makes evoked release less efficient and less synchronous (Mochida et al., 1996; Rettig et al., 1997).

\section{REFERENCES}

Alder J, Lu B, Valtorta F, Greengard P, Poo M (1992) Calciumdependent transmitter secretion reconstituted in Xenopus oocytes: requirement for synaptophysin. Science 257:657-661.

Atwood HL, Govind CK, Wu CF (1993) Differential ultrastructure of synaptic terminals on ventral longitudinal abdominal muscles in Drosophila larvae. J Neurobiol 24:1008-1024.

Barnett DW, Liu J, Misler S (1996) Single-cell measurements of quantal secretion induced by alpha-latrotoxin from rat adrenal chromaffin cells: dependence on extracellular $\mathrm{Ca}^{2+}$. Pflügers Arch 432:1039-1046.

Betz WJ, Bewick GS (1992) Optical analysis of synaptic vesicle recycling at the frog neuromuscular junction. Science 255:200-203.

Betz WJ, Bewick GS (1993) Optical monitoring of transmitter release and synaptic vesicle recycling at the frog neuromuscular junction. J Physiol (Lond) 460:287-309.

Betz WJ, Wu LG (1995) Synaptic transmission: kinetics of synapticvesicle recycling. Curr Biol 5:1098-1101.

Betz WJ, Mao F, Bewick GS (1992) Activity-dependent fluorescent staining and destaining of living vertebrate motor nerve terminals. J Neurosci 12:363-375.

Betz WJ, Mao F, Smith CB (1996) Imaging exocytosis and endocytosis. Curr Opin Neurobiol 6:365-371.

Braun JEA, Scheller RA (1995) Cysteine string protein, a DnaJ family member, is present on diverse secretory vesicles. Neuropharmacology 34:1361-1369.

Braun J, Wilbanks SM, Scheller RH (1996) The cysteine string secretory vesicle protein activates Hsc70 ATPase. J Biol Chem 271:25989-25993.

Broadie K, Prokop A, Bellen HJ, O'Kane CJ, Schulze KL, Sweeney ST (1995) Syntaxin and synaptobrevin function downstream of vesicle docking in Drosophila. Neuron 15:663-673.

Buchner E, Gundersen CB (1997) The DnaJ-like cysteine string protein and exocytotic neurotransmitter release. Trends Neurosci 20:223-227.

Capogna M, Gahwiler BH, Thompson SM (1996) Presynaptic inhibition of calcium-dependent and -independent release elicited with ionomycin, gadolinium, and alpha-latrotoxin in the hippocampus. J Neurophysiol 75:2017-2028.

Ceccarelli B, Grohovaz F, Hurlbut WP (1979) Freeze-fracture studies of frog NMJs during intense release of neurotransmitter. I. Effects of black widow spider venom and $\mathrm{Ca}^{2+}$-free solutions on the structure of the active zone. J Cell Biol 81:163-177.

Chamberlain LH, Burgoyne RD (1996) Identification of a novel cysteine string protein variant and expression of cysteine string proteins in non-neuronal cells. J Biol Chem 271:7320-7323.

Chamberlain LH, Burgoyne RD (1997) Activation of the ATPase activity of heat-shock proteins $\mathrm{Hsc} 70 / \mathrm{Hsp} 70$ by cysteine-string protein. Biochem J 322:853-858.

Chamberlain LH, Henry J, Burgoyne RD (1996) Cysteine string proteins are associated with chromaffin granules. J Biol Chem 271:19514-19517.

Clark AW, Mauro A, Longenecker HJ, Hurlbut WP (1970) Effects of black widow spider venom on the frog neuromuscular junction. Effects on the fine structure of the frog neuromuscular junction. Nature 225:703-705.

Cyr DM, Langer T, Douglas MG (1994) DnaJ-like proteins: molecular chaperones and specific regulators of Hsp70. Trends Biochem Sci 19:176-181.
Fritz LC, Atwood HL, Jahromi SS (1980) Lobster neuromuscular junctions treated with black widow spider venom: correlation between ultrastructure and physiology. J Neurocytol 9:699-721.

Grigliatti TA, Hall L, Rosenbluth R, Suzuki DT (1973) Temperaturesensitive mutations in Drosophila melanogaster. XIV. A selection of immobile adults. Mol Gen Genet 120:107-114.

Gundersen CB, Umbach JA (1992) Suppression cloning of the cDNA for a candidate subunit of a presynaptic calcium channel. Neuron 9:527-537.

Heckmann M, Adelsberger H, Dudel J (1997) Evoked transmitter release at neuromuscular junctions in wild-type and cysteine string protein null mutant larvae of Drosophila. Neurosci Lett 228:167-170.

Henkel AW, Betz WJ (1995) Monitoring of black widow spider venom (BWSV) induced exo- and endocytosis in living frog motor nerve terminals with FM1-43. Neuropharmacology 34:1397-1406.

Heuser JE, Reese TS (1973) Evidence for recycling of synaptic vesicles at the frog neuromuscular junction. J Cell Biol 57:315-344.

Ikeda K, Ozawa S, Hagiwara S (1976) Synaptic transmission reversibly conditioned by a single gene mutation in Drosophila melanogaster. Nature 259:489-491.

Jan LY, Jan YN (1976) Properties of the larval neuromuscular junction in Drosophila melanogaster. J Physiol (Lond) 262:189-214.

Johansen J, Halpern ME, Johansen KM, Keshishian H (1989) Stereotypic morphology of glutaminergic synapses in identified muscle cells of Drosophila larvae. J Neurosci 9:710-725.

Kohan SA, Pescatori M, Brecha NC, Mastrogiacomo A, Umbach JA, Gundersen CB (1995) Cysteine string protein immunoreactivity in the nervous system and adrenal gland of rat. J Neurosci 15:6230-6238.

Kosaka I, Ikeda K (1983a) Possible temperature-dependent blockage of synaptic vesicle recycling induced by a single gene mutation in Drosophila. J Neurobiol 14:207-225.

Kosaka I, Ikeda K (1983b) Reversible blockage of membrane retrieval and endocytosis in the garland cell of the temperature-sensitive mutant of Drosophila melanogaster, shibirets1. J Cell Biol 97:499-507.

Linial M, Ilouz N, Feinstein N (1995) Alpha-latrotoxin is a potent inducer of neurotransmitter release in Torpedo electric organ-functional and morphological characterization. Eur J Neurosci 7:742-752.

Magazanik LG, Fedorova IM, Kovalevskaya GI, Pashkov VN, Bulgakov OV, Grishin EV (1992) Selective presynaptic insectotoxin (alphalatroinsectotoxin) isolated from black widow spider venom. Neuroscience 46:181-188.

Martin-Moutot N, Charvin N, Leveque C, Sato K, Nishiki T, Kozaki S, Takahashi M, Seager M (1996) Interaction of SNARE complexes with $\mathrm{P} / \mathrm{Q}$-type calcium channels in rat cerebellar synaptosomes. J Biol Chem 271:6567-6570.

Mastrogiacomo A, Parsons SM, Zampighi GA, Jenden DJ, Umbach JA, Gundersen CB (1994) Cysteine string proteins-a potential link between synaptic vesicles and presynaptic $\mathrm{Ca}^{2+}$ channels. Science 263:981-982.

Mochida S, Sheng ZH, Baker C, Kobayashi H, Catterall WA (1996) Inhibition of neurotransmission by peptides containing the synaptic protein interaction site of N-type $\mathrm{Ca}^{2+}$ channels. Neuron 17:781-788.

Poodry CA, Edgar L (1979) Reversible alteration in the neuromuscular junctions of Drosophila melanogaster bearing a temperature-sensitive mutation, shibire. J Cell Biol 81:520-527.

Pupier S, Leveque C, Marqueze B, Kataoka M, Takahashi M, Seagar MJ (1997) Cysteine string proteins associated with secretory granules of the rat neurohypophysis. J Neurosci 17:2722-2727.

Ramaswami M, Krishnan KS, Kelly RB (1994) Intermediates in synaptic vesicle recycling revealed by optical imaging of Drosophila neuromuscular junctions. Neuron 13:363-375.

Rettig J, Heinemann C, Ashery U, Sheng Z, Yokoyama CT, Catterall WA, Neher E (1997) Alteration of $\mathrm{Ca}^{2+}$ dependence of neurotransmitter release by disruption of $\mathrm{Ca}^{2+}$ channel-syntaxin interaction. J Neurosci 17:6647-6656.

Ryan TA, Smith SJ (1995) Vesicle pool mobilization during action potential firing at hippocampal synapses. Neuron 14:983-989.

Stanley EF (1993) Single calcium channels and acetylcholine release at a presynaptic nerve terminal. Neuron 11:1007-1011.

Stewart BA, Atwood HL, Renger JJ, Wang J, Wu CF (1994) Improved stability of Drosophila larval neuromuscular preparations in haemolymph-like physiological solutions. J Comp Physiol [A] 175:179-191.

Storchak LG, Pashkov VN, Pozdnyakova NG, Himmelreich NH, Grishin 
EV (1994) Alpha-latrotoxin-stimulated GABA release can occur in $\mathrm{Ca}(2+)$-free, $\mathrm{Na}(+)$-free medium. FEBS Lett 351:267-270.

Sudhof TC (1995) The synaptic vesicle cycle: a cascade of proteinprotein interactions. Nature 375:645-653.

Umbach JA, Gundersen CB (1997) Evidence that cysteine string proteins regulate an early step in the $\mathrm{Ca}^{2+}$-dependent secretion of neurotransmitter at Drosophila neuromuscular junctions. J Neurosci 17:7203-7209.

Umbach JA, Zinsmaier KE, Eberle KK, Buchner E, Benzer S, Gundersen CB (1994) Presynaptic dysfunction in Drosophila csp mutants. Neuron 13:899-907.

Umbach JA, Mastrogiacomo A, Gundersen CB (1995) Cysteine string proteins and presynaptic function. J Physiol (Paris) 89:95-101.

Umbach JA, Grasso A, Mastrogiacomo A, Pescatori M, Buchner E, Güudeisen CB (1995) Soc Neurosci Abstr 21:329. van deGoor J, Kelly RB (1996) Association of Drosophila cysteine string proteins with membranes. FEBS Lett 380:251-256.

Verhage M, McMahon HT, Ghijsen WE, Boomsma F, Scholten G, Wiegant VM, Nicholls DG (1991) Differential release of amino acids, neuropeptides, and catecholamines from isolated nerve terminals. Neuron 6:517-524.

von Gersdorff H, Matthews G (1994) Dynamics of synaptic vesicle fusion and membrane retrieval in synaptic terminals. Nature 367:735-739.

Zinsmaier KE (1997) Cysteine string proteins. In: Guidebook to molecular chaperones and protein-folding catalysts (Gething MJ, ed), pp 115-117. New York: Oxford UP.

Zinsmaier KE, Eberle KK, Buchner E, Walter N, Benzer S (1994) Paralysis and early death in cysteine string protein mutants of Drosophila. Science 263:977-980. 